\title{
Hairy roots characteristics of vegetable pea's morphotype with modified leaf and offspring of the 89/16 sugar line transformant
}

Timina O.O. ${ }^{*}$, Timin O.Y. ${ }^{2}$, Stepanova A.Y. ${ }^{3}$, Solovyeva A.I. ${ }^{3}$

${ }^{1}$ Shevchenko State University, Tiraspol, Moldova, Transnistria

${ }^{2}$ Research Institute of Ecology and Natural Resources, Moldova, Transnistria, Bendery

${ }^{3}$ Timiryazev Institute of Plant Physiology, RAS, Moscow, Russia

*email: otimina@mail.ru

Genetic transformation of the vegetable pea has been tested by three wild strains of Agrobacterium rhizogenes. The steadily growing hairy roots of the acacia-shaped mutant were obtained free from the vector. PCR analysis showed the presence of rol $C$ gene in hairy roots with a total protein content of $46.38 \%$. The amino acid score protein identified seven essential amino acids. Tryptophan was absent in the culture, limiting the fullness of the protein. The vegetable sugar pea transformant was received, the offspring of which was also free from the vector. Plants PCR analysis showed the presence of rol $D$ gene in progeny, including the control variant. It is assumed that the control identified the appropriate homolog as a possible result of natural transformation, which occurred in natural conditions. The offspring of the transformant was characterized by both an increase in overall antioxidant activity and heat resistance in temperature stress $\left(25^{\circ} \leq \mathrm{t} \leq 49^{\circ} \mathrm{C}\right)$, which was assessed by direct method for the absence of leaves necrosis in comparison with control. Transformant's offspring was characterized by smaller leafs sizes and a decrease in the stem length, the lower bean height, the number of knots up to the lower bean and the dry mass of the plant. The results indicate an increase in the rate of reaction of the transformant offspring, which has become consistent with the mesothermophyte (heat-loving) ecogroup in comparison with control. This adaptation may be based on the active expression of the rol $D$ gene, which is known to encode an enzyme that synthesizes proline, an amino acid with antioxidant, anti-denature osmoregulating properties and membrane-protective properties that adapt to stress. It is assumed that the mechanism of increasing the antioxidant activity of transformants is associated with an increase in the number of copies of the rol $D$ gene. 